\title{
Agility Through Design - The Holonic Multi-cell Control System (HoMuCS) Architecture
}

\author{
J. Schnell, G. Langer, C. Sørensen \\ Department of Manufacturing Engineering - DTU, Denmark \\ Em: js@ipt.dtu.dk, gilad@ipt.dtu.dk, cs@ipt.dtu.dk
}

Keywords Holonic manufacturing systems, capabilities, machine integration

\begin{abstract}
In a Holonic system the issues concerned with interfacing to the physical equipment are of utmost importance. The two performance criteria for agile systems defined as structural and operational performance have to be optimised. This necessitates a clear model of the capabilities of the physical equipment encapsulated within the Holon. This is achieved through a generic model representing the states and capabilities of the physical equipments, which the Holon uses for structural and operational changes. This model is implemented in the HoMuCS architecture, which is a specific implementation of the Holonic Manufacturing System theory, with the VMD class. The paper briefly describes the Holonic Manufacturing Systems (HMS) and the HoMuCS architecture. It then supplements with a more in depth description of the characteristics and architecture of this VMD and its capability model. The use of a VMD class is exemplified through a case study.
\end{abstract}

\section{INTRODUCTION}

With the extensive growth and extent of information technology into the manufacturing domain and the growing need of manufacturers to increase the flexibility of their manufacturing facilities, new paradigms for manufacturing are becoming more attractive. One of these new paradigms is the Holonic Manufacturing System theory proposed by the HMS consortium [1]. It presents a solution for manufacturing systems that has the ability to accommodate the increasingly dynamic characteristics of the manufacturing environment. This involves many novel features that need to be considered one of them being the manufacturing system and its control, commonly known as Shop Floor Control (SFC).

Current research at the Department has resulted in the Holonic Multicell Control System concept that consists of a methodology and system- 
architecture [2]. HoMuCS is still under development and this paper describes some of the on going research in the integration of the physical manufacturing equipment.

\section{HOLONIC MANUFACTURING SYSTEMS}

The Holonic Manufacturing System (HMS) concept has evolved as a result of research performed during the last decade. It is inspired by the work of the Hungarian sociologist Athur Köstler. In his book 'The Ghost in the Machine' [3], he proposes a method to describe human behavior and social structures. He introduces the term 'Holon' to describe the basic element of the social structure e.g. the individual. The term 'holon' is made up from the Greek word 'holos' meaning whole and 'on' as in electron that is 'part of' a whole - the atom. The structures that the holons create is called holarchies and are neither just hierarchic nor heterarchic but can be anything in between.

\subsection{HOMUCS}

HoMuCS is acronym for 'Holonic Multi-cell Control System' and names the research in future manufacturing systems carried out at Department of Manufacturing Engineering at the Technical University of Denmark. HoMuCS proposes an architecture and methodology that can be used to engineer manufacturing execution - or shop floor control systems based on the HMS paradigm.

\subsubsection{The architecture}

The HoMuCS engineering concept consists of four elements as shown in Figure 1. The central element here is the architecture, which is the set of customizable components for the Holons. These components are abstract meaning that they have to be customized, as part of the engineering process in order to obtain the holons and other structural elements needed for implementation. These are supported by functional requirements defined by the functional models. Each Holon in the architecture describes a generic component of a shop floor control system. The complete description of the architecture and corresponding models are given in [2].

At the moment the system architecture is implemented using the Java programming language and can be viewed in more detail on the HoMuCS website (http://www.homucs.org). The fundamental objective of the architecture is to provide design simplicity and quality of the engineering of industrial HoMuCS solutions. 


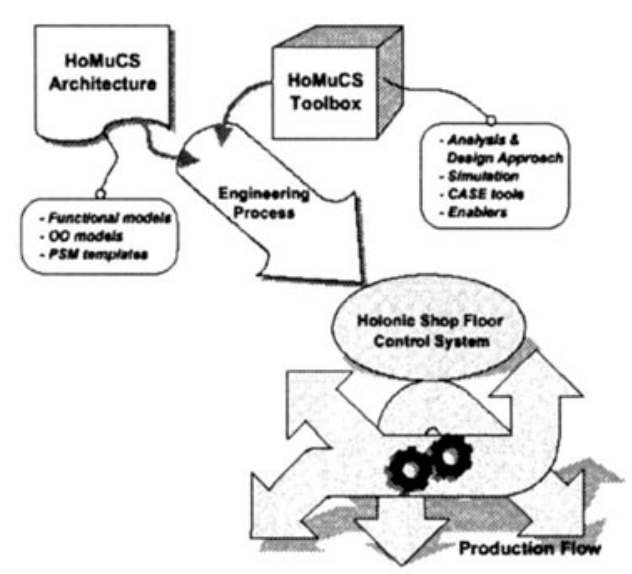

Figure 1: The HoMuCS engineering concept.

\subsubsection{The HoMuCS Methodology}

Implementation of the architecture is performed according to the HoMuCS methodology [2]. The basic idea is that the architecture is used as a set of guidelines and customizable building blocks that have to be specified and extended for implementation of a HoMuCS. The architecture's functional models are used during the analysis phase of the methodology as functional guidelines for a system implementation. Thus during the analysis phase they are extended to include the needed description for implementation of specific Holon functionality that is not included in their generic form given by the architecture.

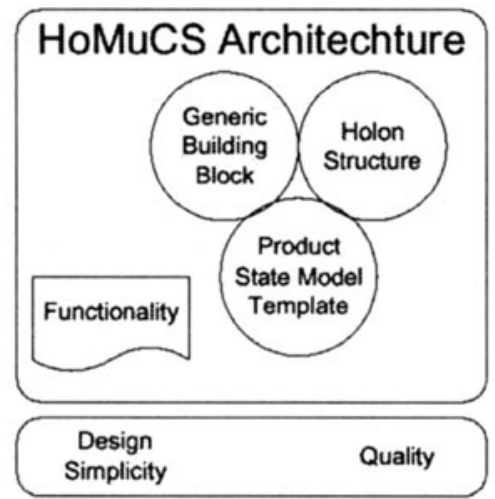

Figure 2: Overview of the HoMuCS architecture

The methodology aids the development of the HoMuCS by directing the assembly of specialization of the abstract components of the architecture. In this way the holonic characteristics of a HoMuCS are secured. 


\subsection{The structure of the Holon}

The HoMuCS architecture defines a resource holon as an abstraction of some production equipment ranging from single machines and plc's to cells, shops and factories. In the context of this paper the main interest is the structure of this types of Holons.

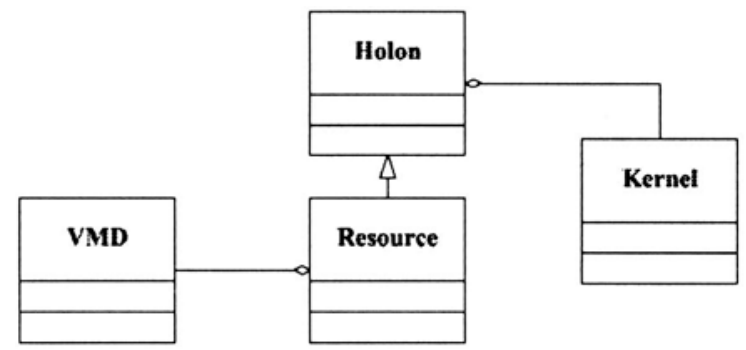

Figure 3 UML structure of the Holon class

A Resource Holon aggregates two main objects, the Kernel and the VMD, Figure 3. Figure 3The Kernel contains the holon's decision logic, which is used to interact using both long and short-term interaction. The Kernel links the holon to the other holons in the holarchy and gives the holon its co-operative behavior. The VMD is the integration of the physical part of the holon. It is the integration of the physical manufacturing equipment that is the main focus of this paper.

\section{Integration with manufacturing equipment}

One important property about holonic manufacturing systems is that the concept, unlike most traditional manufacturing control systems, does not separate the manufacturing system from the manufacturing control system. The implication of this is that it is essential that the actual state and capabilities of the equipment be known to the holon at any time. Thus a Resource Holon has to be able to specify what tasks it is capable of performing. Such information is an essential requirement during the dynamic process of resource allocation in a HoMuCS.

The physical properties of a machine or machine tool determine the capabilities of that resource. Furthermore the required processing function is a synthesis of two or more basic capabilities. For example when a part has to be moved between locations by a robot. The robot consists of a mechanism and a gripper, where the gripper holds the part that has to be moved and the robot mechanism moves the gripper and the part in space. Here the gripper determines the size of the object that could be moved and the robot 
mechanism determines the weight and the distance that artifacts can be moved. Thus in a HoMuCS a Robot Holon requires this information to evaluate whether it is capable of executing the task, which it assesses based on the state and capabilities of the physical robot that it encapsulates.

Previous research has shown the benefits of creating a generic interface to the physical equipment [4]. Such an interface, implemented as a software component, gives access to the physical functions of the manufacturing device. Moreover it solves the problem regarding the access to the state and capabilities of the physical equipment. In the HoMuCS architecture this component is defined as the VMD. The name VMD is inspired by the Manufacturing Message Specification, MMS (ISO 9506) [5], and is an acronym for Virtual Manufacturing Device.

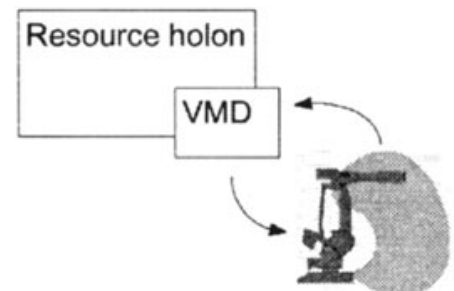

Figure 4 The VMD connects the software and hardware systems

Apart from enabling access to various functions of the equipment, the VMD has a mechanism to evaluate and communicate the capabilities of the equipment. In other words the VMD provides the holon with a generic view and interpretation of the physical equipment that is an integrated part of it. In order do so it has to be able to acquire all the information from the equipment such as states, functions, limitations, etc. and present them as a model of capabilities to the Holon. The holon in turn uses this in its execution logic.

The process of modelling and presenting these capabilities is the main area of focus of this specific research. Currently the capabilities are communicated as text strings that the kernel recognizes and maps them to processing and handling tasks in the manufacturing system.

The VMD performs as a transparent layer between the physical system and the software part of the system, Figure 4. Furthermore it inherently allows for development of a HoMuCS without the need to access the real manufacturing system. This is done by implementing a VMD that emulates the functionality of the physical equipment. This enables the development of SFC systems without direct access to the physical machines and the transfer of the developed system onto the real system by merely changing the VMD implementations. 
Currently a prototype implementation of HoMuCS for a steel plate mill is developed that is used to further develop and test the HoMuCS architecture. A brief presentation of this work that focuses on the details regarding integration of the production equipment is given in the following.

\section{THE OSS CASE}

Odense Steel Shipyard (OSS) produces the world largest container ships. The company is one of the few Scandinavian shipyards, which have survived and prospered, in the strong competition of the last decade. As competition is becoming even harder, OSS continuously thrives to improve the efficiency of their processes. One of these improvements is the implementation of a steel plate milling-cell, an alternative process to using flame cutting for the chamfering process of steel plates. A HoMuCS prototype of this cell has been developed and in the following the results and experiences from this work will be described.

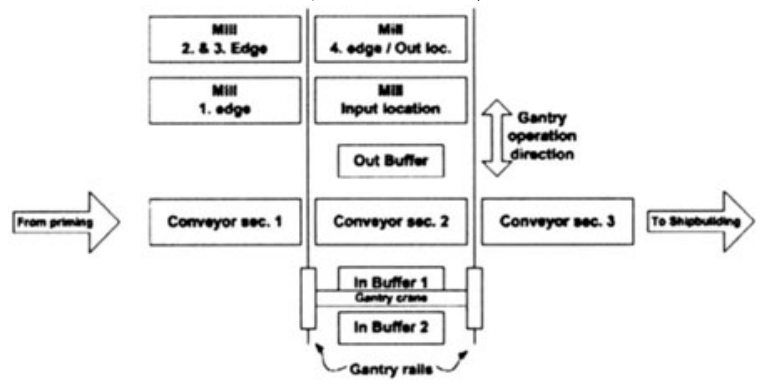

Figure 5 Cell layout

The prototype has a number of holons, three conveyor holons, the crane holon, three buffer holons and the mill holon. Moreover a holon representing the priming station prior to this cell and one representing the shipbuilding after this cell are modeled as wrappers connecting between the holonic cell and the rest of the shipyard.

In this system steel plates are transferred on the conveyors from the priming cell and into the shipbuilding. Some of these plates are assigned for the skin of the ship's hull and need to have their edges chamfered by the milling machine. These plates are lifted off the conveyor and processed by the mill.

In the HoMuCS the steel plate is represented as a plate holon that has to be lifted off the conveyor. To fulfill this requirement a resource holon, with the necessary capabilities that enables it to hold the plate and move it from the conveyor to another location is acquired for that task. The crane's kernel knows through its VMD that it possesses these capabilities and is assigned 
for the task. From the conveyor the plate is routed to the mill that performs the chamfering. However if the mill is not ready to process that plate, the plate is rejected and has to find an alternative location where it can be placed until the mill is ready to process it. The plate has to find a resource that has the capability of storing it for some time. Buffers have this capability and the plate is moved to one of these and placed there until the mill is ready to process the plate.

The order of the plates is prioritized by the due date. An algorithm is used to minimize the chances that plates with lower priority are placed on top of plates with higher priority. The system allows to introduce more buffers can creating locations that have the capability of storing the steel plates. Since the real manufacturing cell was not available during the development of the HoMuCS an emulation model of the cell was built using a standard simulation software package. Emulation is a representation of an object or system with respect to behavior and operation. For example a processing machine on a shop floor is emulated by representing its behavior when initiated externally by a control system or a human operator. The simulation software used allowed for multiple socket-connections and thus a connection was created to every element/holon in the cell. Figure 6 shows the model as it looks in the simulation software.

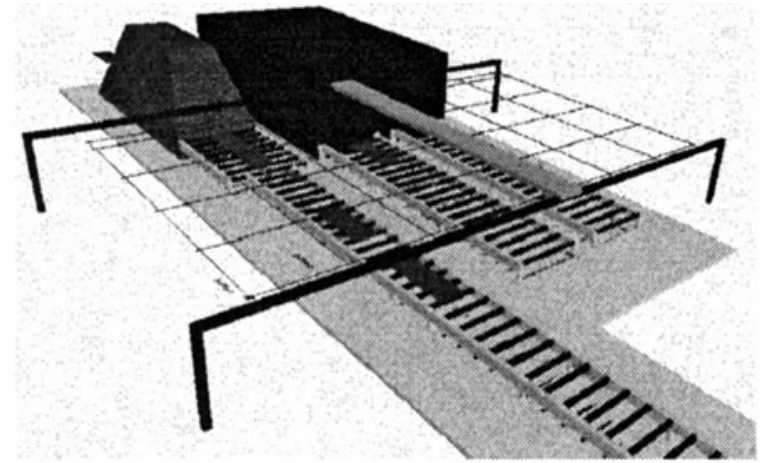

Figure 6 The emulation model

\section{CONCLUSION}

Development of a generic model for representing the capabilities of the Holonic Resources is an essential part of the architecture since it defines the set of tasks that resources are capable of executing. This is vital for Holonic resources since it allows for holon to make their own plans and execute them - or in other word to be autonomous. The use of the VMD, as shown in this paper, is a potentially good way of solving the interface problem in a holonic architecture. Not only does it give a generic interface but serves as the base 
for the implementation of the capabilities model. As the paper points out the actual capability model for a resource still needs much more research. Moreover the mechanism and process where the VMD translates the information from the physical equipment to the generic capability that a Resource Holon can use is still very conceptual and is currently the object of intense research.

Using a generic interface to the physical manufacturing equipment facilitates the integration of the machines that the HoMuCS system is dependent on. These generic interfaces to the physical devices make it easy to reconfigure the device itself and even takeaway the machine and replace it with another. This plug-ability also enables the developer to develop and test the system in a virtual environment before taking the system into the production. These properties are secured by the use of the VMD. The case study was part of the ongoing research effort in the field of HMS, which is dedicated to developing the HoMuCS concept. The author will continue the research around the development of a capability model for the HoMuCS architecture. This will specifically involve a method that enables the Holon to combine capabilities that are for example needed for execution of complex production tasks.

\section{REFERENCES}

[1] Van Brussel, H., Valckanaers, P., Holonic Manufacturing Systems Technical Overview, http://hms.ifw.uni-hannover.de/public/hms_tech.html, PMA Katholieke Universiteit Leuven, Belgium, Dec. 1995.

[2] Langer, G., 1999, HoMuCS - A methodology and architecture for Holonic Multi-cell Control Systems, ISBN 87-90855-00-0

[3] Koestler, A., The Ghost in the Machine, Arkana Books, London 1989, ISBN 0140191925

[4] Schnell, et al., 1999, Development of a robot holon using an open modular controller, Proceedings of 1999 IEEE International Conference on Control Applications, Hawaii, USA

[5] SISCO, 1995, Overview and Introduction to the Manufacturing Message Specification (MMS), Revision 2, Systems Integration Specialists Company, Inc., USA. 